\title{
Photovoltaic energy yield predictions using satellite data
}

Peters, Ian Marius, Liu, Haohui, Buonassisi, Tonio

Ian Marius Peters, Haohui Liu, Tonio Buonassisi, "Photovoltaic energy yield predictions using satellite data," Proc. SPIE 11366, Photonics for Solar Energy Systems VIII, 1136609 (1 April 2020); doi: 10.1117/12.2557375

SPIE. Event: SPIE Photonics Europe, 2020, Online Only 


\title{
Photovoltaic Energy Yield Predictions using Satellite Data
}

\author{
Ian Marius Peters*a, Liu Haohui ${ }^{\mathrm{b}}$, Tonio Buonassisi ${ }^{\mathrm{c}}$ \\ ${ }^{a}$ Forschungszentrum Jülich, HI-ERN, Immerwahrstraße 2, 94058 Erlangen, Germany; \\ ${ }^{\mathrm{b}}$ Solar Energy Research Institute of Singapore (SERIS), 7 Engineering Drive 1, Singapore 117574 \\ 'Massachusetts Institute of Technology, 77 Massachusetts Avenue, Cambridge, MA 02139, USA
}

\begin{abstract}
Energy yield is a key metric for evaluating the performance of photovoltaic systems. It describes the total amount of energy generated by a photovoltaic (PV) installation over a given period, typically a year, and depends on physical properties of the solar cell like efficiency, band gap and temperature coefficient, as well as the operating conditions in a given location. Because the response of a solar cell to these conditions varies, two photovoltaic technologies may have a different energy yield, even if their lab efficiency is identical. Predicting energy yield accurately is important to system operators and installers to estimate the technical and economic performance of a PV installation. In this paper, we summarize our findings about satellite based energy yield predictions of solar cells with various technologies.
\end{abstract}

Keywords: Photovoltaics, Energy Yield, Modelling

\section{INTRODUCTION}

Photovoltaic research has one key figure of merit: the power conversion efficiency $\eta$ measured under standard testing conditions (STC). The existence of a single and generally accepted number to characterize the quality of a solar cell been very helpful to the community. Yet, the conversion efficiency is not the only relevant metric when it comes to the performance of solar cells in the field. Standard testing conditions mark one specific point of operation, which is useful to guarantee reproducibility in the lab, but it is an imperfect representation of the variety of conditions a solar cell will see in the field. This aspect is relevant because different solar cell technologies and architectures have different sensitivities to changing operating conditions. This means that two solar cells may have the same measured efficiency, yet may still generate different amounts of electricity in the field. A quantity that covers the influence of changing conditions is the energy yield - the cumulative power output of a PV installation over a given period. Estimating this number is key to determine the economic performance and return of investment of any PV installation.

Calculating energy yield requires knowing the operating conditions for a solar cell in a given location and for the intended period. Accessing this information is one of the major challenges in calculating energy yield. Accurate yield results require meteorological data with high spatial and temporal resolution, ideally measured on the ground. This information is available for certain locations, but for the majority of regions on the planet, such high quality data sets do not exist. An alternative is the use of satellite-based data. Meteorological information with global coverage over many years is available from different sources. The downside of satellite data is that spatial and / or temporal resolution is often limited, and that values for data on the ground are often calculated rather than directly measured, introducing additional uncertainty. Consequently, using satellite data will result in energy yield estimates with limited precision.

In this paper, we discuss our approach to calculating energy yield based on satellite and ground-based data, and we show a selection of results obtained with this method. Initially, we describe the steps necessary to perform the required calculations and the tools we used to perform them. Being able to do estimates on a global scale allows comparing advantages and disadvantages of photovoltaic technologies. We show how different bandgaps play a role here by comparing performance ratios of cadmium telluride (CdTe) and silicon $(\mathrm{Si})$ solar cells. Finally, we explore the impact of variations in operating conditions on the performance of tandem solar cells compared to single junction solar cells using the example of GaAs on Si two terminal and four terminal tandems.

Photonics for Solar Energy Systems VIII, edited by Alexander N. Sprafke, Jan Christoph Goldschmidt, Gregory Pandraud, Proc. of SPIE Vol. 11366, 1136609 - (c) 2020 SPIE

CCC code: $0277-786 \mathrm{X} / 20 / \$ 21 \cdot$ doi: $10.1117 / 12.2557375$ 


\section{METHOD}

The operating conditions relevant to predicting the performance of a given solar cell are the irradiance $I$, the form of the spectrum $\Phi(\lambda)$ and the junction temperature $T_{\mathrm{j}}$. With the exception of $I$, these values are typically unknown and need to be calculated.

\subsection{Spectrum Calculations}

A number of methods exist to calculate the spectral distribution at a given location and for given environmental parameters. We have used the SMARTS method, developed by C. Gueymard [1,2]. In SMARTS, the solar spectrum is calculated based on a number of input parameters, including irradiance, ambient temperature $T_{\mathrm{a}}$, total precipitable water, aerosol parameters including, aerosol optical depth (AOD), single scattering albedo and angstrom coefficient, as well as ground albedo. These parameters are available publicly from the Earth Observatory Database from NASA. Irradiance data were taken from NASA's Clouds and Earth's Radiant Energy System (CERES) satellite instrument. Total precipitable water, relative humidity at the surface and air temperature were taken from NASA's Atmospheric InfraRed Sounder instrument from the Earth Observing System. Aerosol parameters, as well as ground reflectance data were taken from NASA's Ozone Monitoring Instrument and from NASA's Moderate Resolution Imaging Spectroradiometer. The spatial resolution of all datasets was scaled to $1 \mathrm{x} 1$ degree, the temporal resolution to one point per day. If a higher spatial or temporal resolution was available, averages were calculated.

The limited temporal resolution meant that only one spectrum per day could be calculated using average conditions. Where possible, data was used that was measured at conditions around noon, because it was assumed that these conditions would be most relevant for calculating yield. Comparing simulated and measured spectra showed some discrepancies, yet comparing solar cell performance showed that the main differences in yield between technologies over a year due to variations in spectrum and irradiance could be captured.

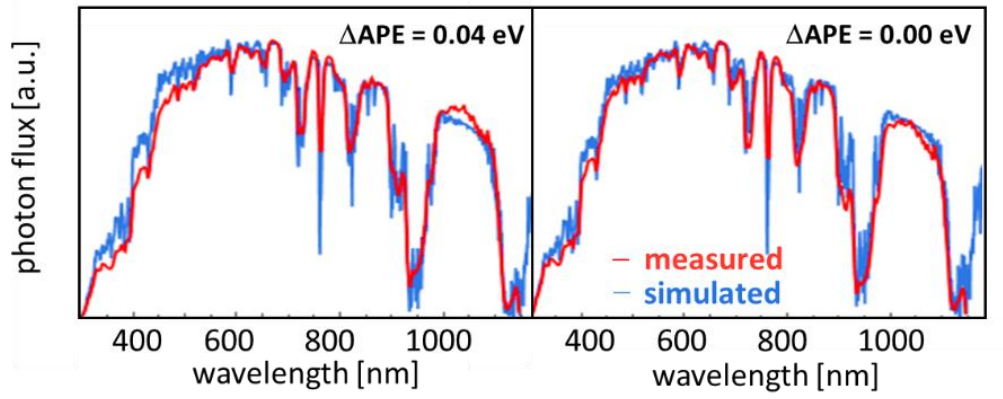

Figure 1. Comparison of simulated and measured spectra. Left, the worst case - the measured spectrum shows a notable blue shift, right the best case with only minor differences. The majority of cases is closer to idea $(\triangle \mathrm{APE}$ mean $=-0.016 \mathrm{eV})$. Generally, there is a good average match in the red part of the spectrum - which is most relevant for comparing performance of different solar cell technologies.

\subsection{Calculating junction temperature}

To calculate the junction temperature $T_{j}$, two coupled equations need to be solved [3]:

$$
\begin{aligned}
& T_{j}=c_{T} \cdot I \cdot\left(\frac{\alpha \cdot \tau}{u_{L}}\right) \cdot\left(1-\frac{\eta_{C}(T)}{\alpha \cdot \tau}\right)+T_{a} \\
& \eta_{C}(T)=\eta_{0}\left(C_{m p p}\left(T_{a}-T\right)+1\right)
\end{aligned}
$$

In these equations, $\alpha$ is the fraction of the solar spectrum absorbed $(0.8), \tau$.the transmittance of glazing $(0,95), u_{L}$ the heat loss coefficient (25) and $T_{a}$ the measured ambient temperature. Numbers in brackets correspond to typical values of a well-ventilated module. $\eta_{C}(T)$ is the temperature dependent efficiency, $\eta_{0}$ the efficiency under standard testing conditions and $C_{m p p}$ the linear temperature coefficient of the solar cell. Because junction temperature and module efficiency are coupled, the equation need to be solved iteratively. 
A particular issue here is the use of a single value for ambient temperature and irradiance. Average daily values are used, yet a module generates the majority of its power at high irradiance conditions around noon. For this purpose, we have introduced a temperature correction factor $c_{T}$ based on detailed data available for Denver and Singapore; we found that a value of 2.6 for this factor provided the closest fit between the detailed, high-resolution data and average conditions based on satellite data.

\subsection{Calculating yield}

To calculate energy yield, first the calculated spectrum needs to be folded with the external quantum efficiency (EQE) of the solar cell, to obtain the short circuit current $\left(j_{\mathrm{SC}}\right)$. With known, $j_{\mathrm{SC}}$ the temperature corrected IV curve for one particular operating condition can be calculated. Several procedures are possible; we used a one-diode representation for a given solar cell to generate an IV curve at standard temperature $\left(25^{\circ} \mathrm{C}\right)$, obtained the maximum power point (MPP) of this curve, and then corrected the power for different temperature using tabulated temperature coefficients. This procedure was repeated once for every day over the ten-year period between 2006 and 2015 and for every grid point on land of the planet.

\section{RESULTS}

\subsection{Year performance Comparison of $\mathrm{CdTe}$ and $\mathrm{Si}$}

Different solar cell technologies have different sensitivities to operating conditions. Differences may originate in the solar cell architectures or in the used materials. One example for an architecture related effect is the range of temperature coefficients found in silicon solar cells. Cells with a higher open circuit voltage tend to have a smaller temperature coefficient than cells with a lower voltage. Even greater differences can be found if the absorber materials are different. Solar cells with a smaller band gap tend to have a greater sensitivity to temperature, as well as being more affected by spectral changes due to precipitable water. The reason for the latter is that water has several absorption bands in the infrared. The smaller the band gap of a solar cell, the further its absorption extends into the infrared, and the more water absorption will reduce the generated current. Conversely, aerosols have a relatively greater effect on solar cells with a larger band gap as they tend to extinct light predominantly in the short wavelength range [4].

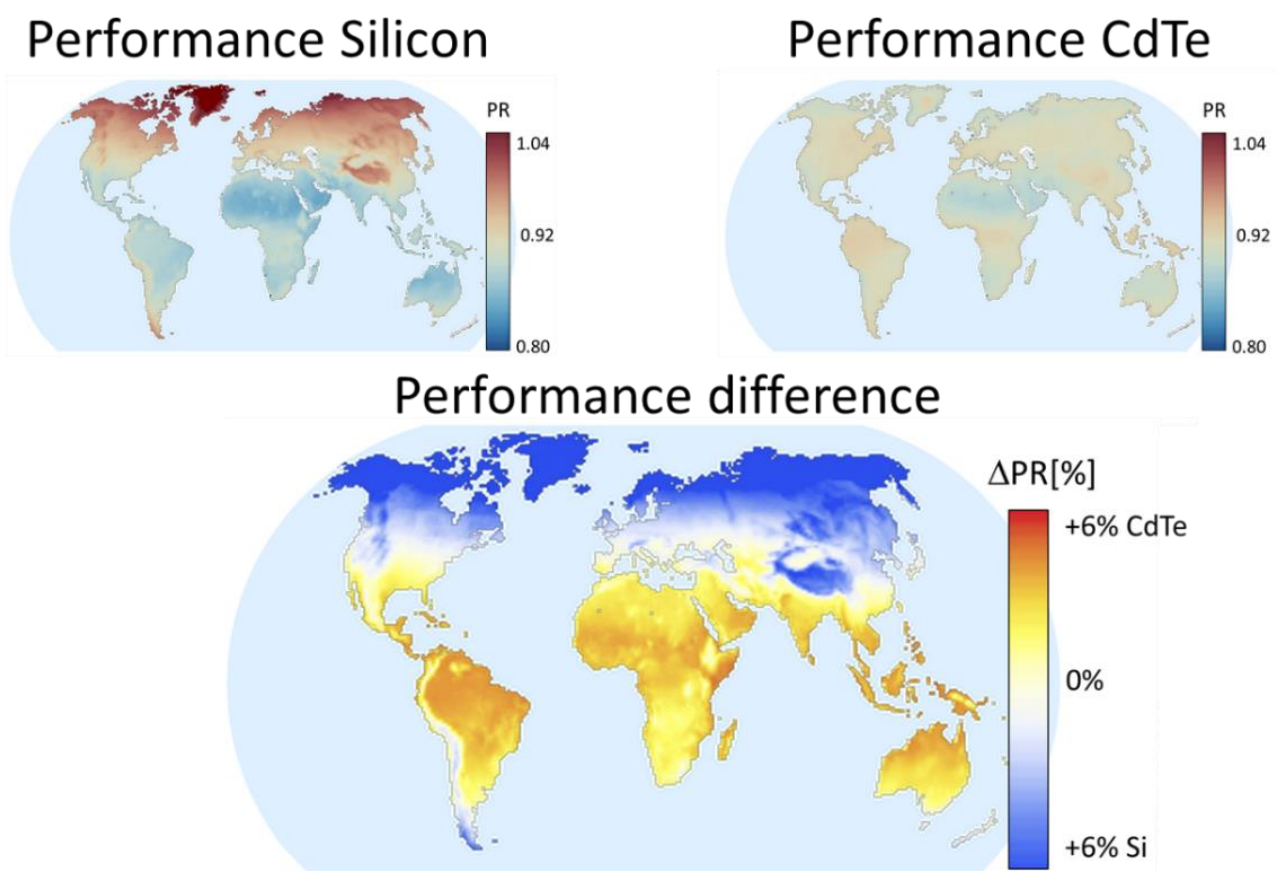

Figure 2. Average performance of silicon (upper left) and CdTe (upper right) solar cells due to discrepancies between lab conditions and actual operating conditions. The portrayed data shows averages obtained over the ten-year period between 2006 and 2015. The lower image shows the performance differences between the two technologies. Blue areas mark a better performance of silicon, red and yellow areas mark a better performance of CdTe. 
A good example for how different operating conditions can result in performance differences are silicon and CdTe solar cells. Silicon has a band gap of $1.12 \mathrm{eV}$, CdTe one of $1.54 \mathrm{eV}$ - with different values being possible depending on the exact material composition. As mentioned, temperature coefficients for silicon vary, in the presented example we are using a value of $-0.45 \% / \mathrm{K}$, corresponding to a PERC type solar cell with $20.7 \%$ efficiency [5]. As an example for a CdTe solar cell, we use the characteristics of a First Solar Series 4 module, with a temperature coefficient of $-0.26 \% / \mathrm{K}$ and an efficiency of $15.6 \%$ [6]. Simulated performance ratios, i.e. energy output compared to output under standard testing conditions over ten years are shown and compared in Figure 2.

The figure illustrates the greater sensitivity of silicon to variation in operating conditions compared to CdTe. Both solar cells generally perform better in higher latitudes and worse in dessert areas (compare Figure 3), but the differences are more distinct for silicon. When comparing the two technologies (lower part of figure 2), we find a correspondence between the climatic condition and performance difference. In cold and dry areas, silicon has a performance advantage. In hot and / or humid areas, CdTe suffers fewer losses than silicon and performs better. The performance difference is synonymous with the relative difference in yield, in case the two solar cell have the same efficiency. The estimated differences in most areas has a magnitude of up to $\pm 6 \%$. We have shown similar results in [7] already for a single year (2015), and find that the overall trends are consistent with the longer-term analysis. Comparing one and ten-year results, we observe a smoothing of the overall trend, especially in the high latitude regions and parts of Africa.

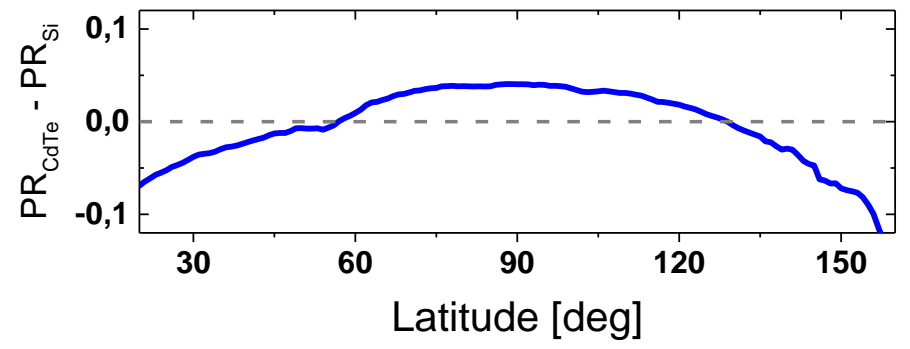

Figure 3. Average performance difference between $\mathrm{CdTe}$ and $\mathrm{Si}$ as a function of latitude.

\subsection{Lessons for Tandem Solar Cells}

Tandem, or more precisely double-junction, solar cells are a particularly interesting case because of the interplay between top and bottom cell performance. Generally, there are two types of architectures for tandem solar cells; two terminal tandems (2T) with an integrated architecture and a tunnel junction, and four terminal (4T) tandems, in which the sub-cells are contacted individually. A number of alternatives also exist that are conceptually similar to one of the two considered concepts and show similar performance. Specific to the $2 \mathrm{~T}$ architecture is the need for current matching between the top and the bottom cell. Tandem performance is quite sensitive to current matching, consequently the question arises how well tandems perform if operating conditions are changing.

Given that tandems are optimized for one particular condition (typically, STC are used), and that with shifts in spectrum either the top or the bottom cell deviate from ideal current generation, one could suspect that tandems are especially sensitive to changing conditions. Yet, this suspicion does not stand when looking at detailed performance modelling. Generally, we find that the performance of $2 \mathrm{~T}$ tandems falls in between the performance of the constituting sub-cells, and $4 \mathrm{~T}$ tandems show a performance that is more similar to the better performing sub-cell. This behavior is shown, exemplarily, in Figure 4 for hypothetical solar cells made of Silicon and GaAs [8]. The figure shows detailed performance calculated for Denver and Singapore, both in the year 2015. We find a similar behavior also for other tandem architectures. In all cases, we found that tandems did not perform worse than single-junction solar cells, but did perform similarly well.

Regarding spectral losses, we found that customization to a given location resulted in a notable improvement in both performance and yield for a solar cell [9]. In Singapore, spectra tend to be blue shifted. Consequently, current generation is shifted to the top cell. Adjusting cell thicknesses resulted in harvesting efficiency improvements of $2 \mathrm{~T} \mathrm{GaAs} / \mathrm{Si}$ tandems of $0.5 \%$, corresponding to about $2 \%$ in performance ratio. Note that this optimization is already included in the results shown in Figure 4. 

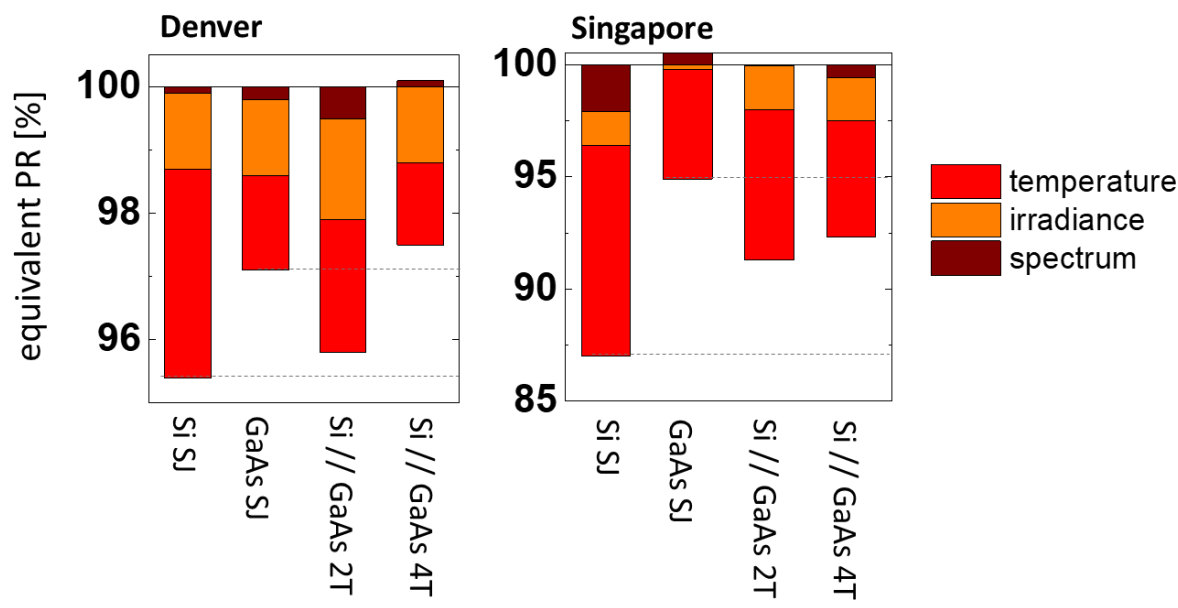

Figure 4. Comparison of the performance ratios of Single Junction Si and GaAs solar cells and two tandem solar cell architectures (2T and 4T) in Singapore and Denver. Also shown is a breakdown into gains and losses due to variations in temperature, irradiance and spectrum.

Several factors contribute to explaining why tandem solar cells perform similarly as single junction solar cells. One factor is the deviation from the ideal in a real tandem solar cell. An ideal tandem considers an ideal band gap pairing with ideal, grey-body absorption. In practice, available band gaps will deviate from the ideal, and absorption is a function of thickness. As conditions deviate from the ideal, the sensitivity to changing conditions is reduced. A second factor is the distribution of performance losses. In the majority of cases, temperature is the most relevant contributor, whereas spectral effects play a relatively minor role (compare figure 4). Temperature affects primarily voltage and fill factor, the impact on current is relatively small. As the voltages of sub-cells in a $2 \mathrm{~T}$ tandem add up, the relative reduction in the $2 \mathrm{~T}$ tandem is always in between those of the single junction cells - this effect is clearly visible when comparing the red portions of the columns in Figure 4.

A third factor is the distribution of the occurring operating conditions. One example for the statistical distribution over one year of temperature, relative humidity and average photon energy [10] (APE) is shown in Figure 5. While occurring conditions may vary over a wide range, the prevalent conditions under which the majority of power is generated lie within relatively small bounds. As an example, occurring spectra had an APE between $1.5 \mathrm{eV}$ and $1.9 \mathrm{eV}$, yet more than $50 \%$ of all power was generated at a value of $1.7 \pm 0.03 \mathrm{eV}$. So, while changing conditions may effect tandems more than single junctions, energy yield is primarily determined by performance under comparably stable conditions.

Temperature

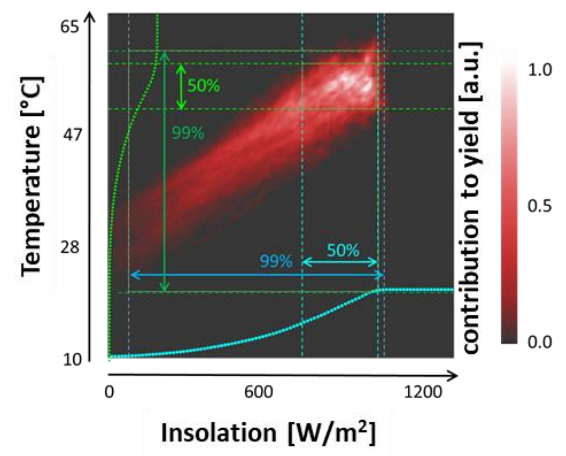

Humidity

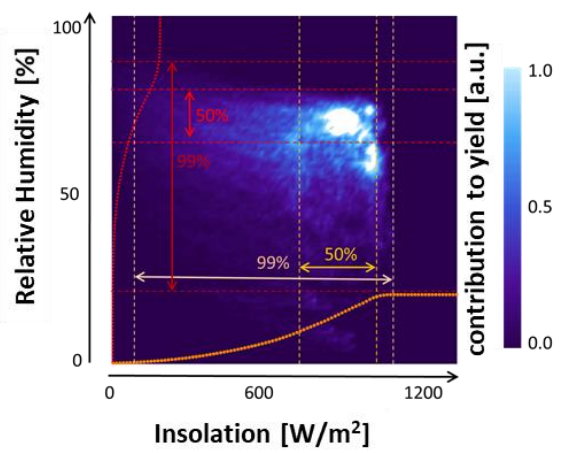

Spectrum

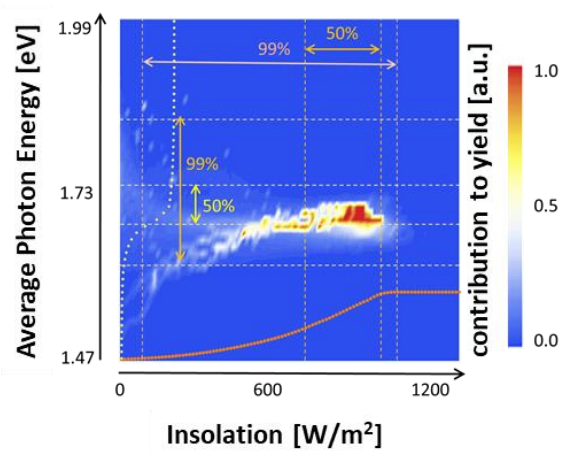

Figure 5. Relation between operating conditions and the contribution of each condition to energy yield. The example shows the case for a hot and arid climate. 


\section{SUMMARY AND DISCUSSION}

Energy yield is an important metric for solar cells and allows technical and economical insights that go beyond those contained in the power conversion efficiency. In this paper, we discuss our approach to perform energy yield calculations based on satellite data and on ground based data. We describe measurement steps and the tools we use to perform them and show a selection of results.

Different solar cells perform differently when exposed to varying operation conditions. As a result, two solar cell may have different energy yields even if they have the same STC efficiency. A significant factor in this difference is the band gap of the used absorber material. Materials with smaller band gaps tend to have lower sensitivities to temperature and spectrum changes than cells with a larger band gap. As an example for two solar cell technologies with different band gaps we explore the case of silicon $(1.12 \mathrm{eV})$ and cadmium telluride $(1.54 \mathrm{eV})$. For these two solar cell technologies, we compare ten-year performance ratios on a global level. In line with expectations, we find that silicon has performance advantages in cold and dry climates, whereas CdTe has advantages in hot and / or humid climates. Plotted over latitude, the performance ratio difference of these two technologies forms an arc with advantages of up to $6 \%$ for each technology in locations relevant for solar cell operation.

Tandem solar cells are another technology for which energy yield considerations are relevant. The combination of two absorber materials with different band gaps generates a particular situation. This is especially the case for the integrated two terminal architecture, in which the sub-cell with lower generation limits the current of the tandem. Because of this limitation, it could be assumed that tandems perform worse than single-junction solar cells. We find that this is not the case; rather does the performance of the $2 \mathrm{~T}$ tandem lie in between those of the single junction solar cells from its constituting materials. 4T tandems have an even better performance - one that is similar to the better performing sub-cell. Possible reasons for this behavior is the prevailing role of temperature compared to other operating conditions as well as the relatively small range of conditions in which most of the energy is generated.

\section{DATA AVAILABILITY}

Insolation data were obtained from the NASA Langley Research Center Atmospheric Science Data Center. Insolation data used in this study is available via NASA's Clouds and the Earth's Radiant Energy System (CERES) website https://ceres.larc.nasa.gov/products.php?product=SYN1deg (registration required). Data can be ordered via the Atmospheric Science Data Center (ASDC) https://eosweb.larc.nasa.gov/project/ceres/syn1deg_table. Temperature, humidity and aerosol data are available from NASA's EARTHDATA website https://search.earthdata.nasa.gov. Temperature and Humidity data using the data set entitled: AIRS/Aqua L3 Daily Standard Physical Retrieval (AIRS-only) 1 degree $\mathrm{x} 1$ degree V006 (AIRS3STD) at GES DISC, aerosol data using OMI/Aura Near UV Aerosol Optical Depth and Single Scattering Albedo L3 1 day 1.0 degree x 1.0 degree V3 (OMAERUVd) at GES DISC (single Scattering Albedo) and MODIS/Terra Aerosol Cloud Water Vapor Ozone Daily L3 Global 1Deg CMG (AOD and Angstrom parameter).

\section{ACKNOWLEDGMENTS}

This work was financially supported by the Project PV-Tera, by the DOE-NSF ERF for Quantum Energy and Sustainable Solar Technologies (QESST) and by funding from Singapore's National Research Foundation through the Singapore MIT Alliance for Research and Technology's "Low energy electronic systems (LEES) IRG”.

\section{REFERENCES}

[1] Gueymard, C.A. "SMARTS, A Simple Model of the Atmospheric Radiative Transfer of Sunshine: Algorithms and Performance Assessment". Professional Paper FSEC-PF-270-295. Florida Solar Energy Center, 1679 Clearlake Rd., Cocoa, FL 32922 (1995).

[2] Gueymard, C.A.. "Parameterized transmittance model for direct beam and circumsolar spectral irradiance." Sol. Energy 71 (5), 325-346 (2001).

[3] Skoplaki, E., Palyvos, J.A., "Operating temperature of photovoltaic modules: a survey of pertinent correlations", Renew. Energy, 34, 23-29 (2009).

[4] Peters, I. M., Karthik, S., Liu, H., Buonassisi, T., Nobre, A.,"Urban haze and photovoltaics", Energy \& Environmental Science 11, 3043-3054 (2018). 
[5] http://www.firstsolar.com/-/media/First-Solar/Technical-Documents/Series-4-Datasheets/Series-4V2Datasheet.ashx.

[6] Fell, A., McIntosh, K., Altermatt, P., "Input parameters for the simulation of silicon solar cells in 2014", IEEE JPV 5, 1250-1263 (2015).

[7] Peters, I. M., Liu, H., Reindl, T., Buonassisi, T., "Global prediction of photovoltaic field performance differences using open-source satellite data", Joule 2 (2018), 307-322.

[8] Liu, H., Ren, Z., Liu, Z., Aberle, A. G., Buonassisi, T., Peters, I. M., „The realistic energy yield potential of GaAson-Si tandem solar cells: a theoretical case study", Optics express 23 (7), A382-A390 (2015).

[9] Liu, H., Aberle, A. G., Buonassisi, T., Peters, I. M., "On the methodology of energy yield assessment for oneSun tandem solar cells", Solar Energy 135, 598-604 (2016).

[10] Minemoto, T.,Nakada, Y., Takahashi, H., Takakura, H., "Uniqueness verification of solar spectrum index of average photon energy for evaluating outdoor performance of photovoltaic modules", Solar Energy 83, 1294 1299 (2009). 